INPE-9879-PRE/5454

CHARACTERISTICS AND APPLICATIONS OF THE NEW HIGH LEVEL ARCHITECTURE-HLA TO PARALLEL OR DISTRIBUTED SIMULATION AND CONTROL OF AEROSPACE VEHICLES

Gilberto da Cunha Trivelato* Marcelo Lopes de Oliveira e Souza

* EMBRAER -Empresa Brasileira de Aeronáutica

Paper presented at the AIAA Modeling and Simulation Technologies Conference, 11-14 Aug 2003, Austin, Texas, USA. 


\title{
CHARACTERISTICS AND APPLICATIONS OF THE NEW HIGH LEVEL ARCHITECTURE - HLA TO PARALLEL OR DISTRIBUTED SIMULATION AND CONTROL OF AEROSPACE VEHICLES
}

\author{
Gilberto da Cunha Trivelato, M.Sc. \\ EMBRAER - Empresa Brasileira de Aeronáutica S. A. \\ P.O. Box 8050 \\ 12227-901 - S. José dos Campos - SP - Brasil \\ trivelato@embraer.com.br \\ Marcelo Lopes de Oliveira e Souza, Ph.D. \\ INPE - National Institute for Space Research \\ DMC - Division of Space Mechanics and Control \\ Av. dos Astronautas, 1758 \\ 12227-010 - S. José dos Campos - SP - Brasil \\ marcelo@dem.inpe.br
}

\begin{abstract}
In this work we discuss the characteristics and applications of the New High Level Architecture-HLA to parallel or distributed simulation and control of aerospace vehicles. HLA is a new standard that is being developed since 1996 to replace previous standards as the Distributed Interactive Simulation -DIS standard in the tasks of interconnecting the main elements of parallel or distributed simulation and control through a common Run Time Infrastructure-RTI. HLA is being developed under the technical guidance and administrative leadership of the Defense Modeling and Simulation Office - DMSO of the Department of Defense - DOD of USA. Since 1998 DOD imposed successive (no pay-1998, no play-1999) deadlines to the defense industry for ending the use of previous standards and beginning the use of HLA. Since then and due to that, its adoption is growing steadily, and its versions matured rapidly to the point that it became the IEEE 1516 standard for modeling and simulation in 2000. This is the version whose characteristics (Interoperability, Reuse, Connectivity, Open Standard, Run Time Infrastructure, Maturity, Adherence to legacy projects and to object oriented projects, etc.) will be discussed in this work, specially for applications to parallel or distributed simulation and control of aerospace vehicles.
\end{abstract}

KEYWORDS: High Level Architecture, Parallel or Distributed Simulation and Control, Real Time Simulation and Control, Aerospace Vehicles

\section{INTRODUCTION}

The Defense Modeling and Simulation Office (DMSO), created in June 1991, is the main Organization for Department of Defense (DoD) modeling and simulation $(M \& S)$ and ensures that $M \& S$ technology development is consistent with other related initiatives. The first results arrives in January 1994, with the publication of Directive 5000.59 "DoD Modeling and Simulation (M\&S) Management", known as the first step to implement an M\&S development policy in DoD.

Using this document (DoD 5000.59-P), in October 1995 the DMSO lead one development group to reach the interoperability among all kind of models and simulations related to C4I (Command, Control, Communication, Computer, and Intelligence). The "Simulation Master Plan" (named M\&S HLA) was developed under the Defense Advanced Research

Copyright $\odot 2003$ by G. C. Trivelato and M. L. O. Souza

Published by The American Institute of Aeronautics

and Astronautics, Inc., with permission.
Projects Agency (DARPA) Advance Simulation Program (ADS). In this program the "simulations need to be interactive through current state-of-the-art communication systems", the main goals were:

- Develop a common technical framework for M\&S. (High Level Architecture, mission conceptual models and data standardization).

- Provide timely and authoritative representations of the natural environment.(terrain,ocean, atmosph.,space).

- Provide authoritative representations of systems.

- Provide authoritative representations of human behavior (individual, groups and organizations).

- Establish an M\&S Infrastructure to meet developer and end-user needs.

- Share the benefits of M\&S. (quality, education and reuse).

American Institute of Aeronautics and Astronautics 
In this work we discuss the characteristics and applications of the new High Level Architecture-HLA to parallel or distributed simulation and control of aerospace vehicles. HLA is a new standard that is being developed since 1996 to replace previous standards as the Distributed Interactive Simulation -DIS standard in the tasks of interconnecting the main elements of parallel or distributed simulation and control through a common Run Time Infrastructure-RTI. HLA is being developed under the technical guidance and administrative leadership of the Defense Modeling and Simulation Office - DMSO of the Department of Defense - DOD of USA. Since 1998 DOD imposed successive (no pay-2000, no play-2001) deadlines to the defense industry for ending the use of previous standards and beginning the use of HLA. Since then and due to that, its adoption is growing steadily, and its versions matured rapidly to the point that it became the IEEE 1516 standard for modeling and simulation in 2002. This is the version whose characteristics (Interoperability, Reuse, Connectivity, Open Standard, Run Time Infrastructure, Maturity, Adherence to legacy projects and to object oriented projects, etc.) will be discussed in this work, specially for applications to parallel or distributed simulation and control of aerospace vehicles.

\section{HLA OVERVIEW}

The complete HLA standard is presented in the IEEE Std $1516^{1}$, IEEE Std $1516.1^{2}$ and IEEE Std 1516.2 ${ }^{3 .}$ For those ones that need a brief presentation a good reference introduction is presented in Kuhl et alli ${ }^{4}$.

According the standard "The High Level Architecture (HLA) is an integrated architecture that has been developed to provide a common architecture for $M \& S$ ". The HLA is based on federates and federations:

"Federate: All applications participating of an HLA federation." Individual federates can be simulations, supporting utilities, or interfaces to live systems.

"Federation: Interfacing federates, common federation object model and supporting RTI used for some specific objective." Federation is a set of federates.

The three main components are: Framework and Rules, Object Model Template (OMT) Specification, and Federate Interface Specifications (IS). The resume for de HLA is following presented.

Framework and Rules

The framework and rules document " "provides an overview of the High Level Architecture (HLA), defines a family of related HLA documents, and defines de principles of HLA in terms of responsibilities that federates (simulations, supporting utilities, or interfaces to live systems) and federations (sets of federates working together) must uphold". It defines de general principles for federates and federations achieve proper integration in run time. It contains five rules that apply to federations and five rules that apply to federates.

\section{Object Model Template (OMT) Specification}

The framework and rules document" "defines the format syntax for recording information in High Level Architecture (HLA) object models, attributes, interactions, and parameters. It does not define the specific data (e.g., vehicles, unit types) that will appear in the object models". It defines de general principles for federates and federations achieve proper integration in run time. "The OMT consists of the following components (from the standard ${ }^{2}$ ):

Object model identification table: To associate important identifying information with the HLA object model.

Object class structure table: To record the namespace of all federate or federation object classes and to describe their class-subclass relationships.

Interaction class structure table: To record the namespace of all federate or federation interaction classes and describe their class-subclass relationships.

Attribute table: To specify features of objects attributes in a federate or federation.

Parameter table: To specify features of interaction parameters in a federate or federation.

Dimension table: To specify dimensions for filtering instance attributes and interactions.

Time representation table: To specify the representation of time values.

User-supplied tag table: To specify the representation of tags used in HLA services.

Synchronization table: To specify representation and datatypes used in HLA synchronization services.

Transportation type table: To specify the transportation mechanisms used.

Switches table: To specify initial settings for parameters used by RTI.

Datatypes table: To specify details of data representation in the object model.

Notes table: To expand explanations of any OMT tables item.

FOM/SOM lexicon: To define all the objects, attributes, interactions, and parameters used in the HLA object model." 
Federate Interface Specifications (IS)

The interface specification" "defines the interface between federates (simulations, supporting utilities, or interfaces to live systems) and the underlying software services that support interfederate communication in a distributed simulation domain”. It specifies the standard services and interfaces between federates and Run Time Infrastructure (RTI). The seven service group, management object model and connection to the APIs are described in details in the standard and here we present an abstract from there:

Federation Management

"Federation management refers to creation, dynamic control, modification, and deletion of a federation execution."

Declaration Management (DM)

"Joined federates shall use DM services to declare their intention to generate information".

Object Management

"This HLA services shall deal with registration, modification, and deletion of object instances and the sending and receipt of interactions.“

Ownership Manager

"Ownership management shall be used by federates and the RTI to transfer ownership of instance attributes among joined federates".

Time Management

Time in the system being modeled shall be represented in the federation as points along a federation time axis. "The time management services and mechanisms provide a federation execution with means to order the delivery of messages throughout the federation execution".

Data distribution management (DDM)

"DDM services may be used by joined federates to reduce both the transmission and the reception of irrelevant data".

Support services

"Miscellaneous services utilized by joined federates performing such actions as: name-to-handle and handleto-name transformation, setting advisory switches, manipulating regions, RTI start-up and shutdown", etc.

Management object model (MOM)

"MOM provide facilities for access to RTI operating information during federation execution". It is used to provide insight the operations of federates and the RTI and to control the functioning of the RTI.

Support services

This clause provides the reader with a mapping from abstract view to the programming languages specific views in the presented APIs.
RunTime Infrastructure (RTI)

"The general purpose distributed operating system (layer) software that provides the common interface services during the runtime of a High Level Architecture federation." The RTI implementation basically contains:

"Federation executive: a global process of each separate federation execution, witch controls joining and resigning of federates, and assures proper FOM data exchange."

"RTI Executive: a global process for the creation, destruction, location, and connection to federations executions."

"RTI federate library: a software library implementing the RTI interfaces service."

In order to set up and run a federation execution the RTI needs: i) a federation execution data (FED), a standard file exchange format that shall be used to store and transfer HLA FED files between multiples tools including object-model development tools and RTI, and ii) initialization data file containing the information to control RTI software.

\section{ENVIRONMENTS FOR SIMULATION AND CONTROL OF AEROSPACE VEHICLES}

Since its beginning, the aerospace industry became one of the main users and beneficiaries of simulation, stimulating and permitting the development of tools even more powerful. Successively, it took advantage of the use of: physical simulation for design and training; simulation in analog computers for control systems; and, in the age of digital computers, digital simulation in the remaining areas of knowledge involved in the diverse phases of development of aerospace vehicles. Today, simulation is recognized even more as an essential tool for the diverse phases of product such as: specification, design, development, production, tests, accreditation, maintenance, training, and marketing.

The complexity of new aerospace vehicles and its present and future applications (always determining the reduction of costs, less weight and consumption, for optimizing such designs) and the need of reducing the production cycle, created new problems of distinct nature, that did not exist or that were neglected before. We can not face these problems without the support of simulation and rapid prototyping, including those simulations with hardware, pilots in the loop or even real players.

For the adequate use of simulation in the development of an aerospace vehicle or defense system it is necessary to observe the important aspects of each phase with respect to those environments. The current 
designs in this area may be developed by teams distributed even in diverse places around Earth, which requires standardization and universality as a necessary requirement for the specification of systems, to have the same meaning among the diverse teams.

The interoperability and reuse of models are essential for prototyping the systems under diverse technical aspects and in diverse places during the development phase, and to allow adequate environments for the integration and tests of subsystems. It also became fundamental the reuse of models of the diverse systems along the diverse phases of the project as a way of reducing time and costs, increasing the reliability, the ease of accreditation, the fidelity for training, the reproduction and investigation of faults or accidents, etc. All these prototypes, in the whole or in part, can and must be used for the safety assessment and accreditation of the product under the correspondent institutions (FAA, JAA, CTA, etc.), for training the members involved during the program, and for training the customers in the operation and maintenance of the final product. It must be possible the reuse and incorporation of legacy codes that were already verified, validated and accredited. With the inclusion of these requirements in the beginning of the specification phase, we have in the end truly simulated systems available for the investigation of failures and for maintenance of the product developed. The simulation software can be used for marketing or for support to the sales during product development and in the after-sale. The use of environments that meet these requirements from product conception to after-sale represents significant savings with benefits in the product reliability and in the reduction in the development time. The use of same standards in synthetic environments is important for the Systems Integration and Tests Area of very complex systems as military aircraft, space shuttles interplanetary trip and defense systems. These standardized environments are becoming de facto standards in the aerospace area due to their dynamic capacity of meeting the ever-growing needs; and from our point of view, they already became essential tools due to the reduction of deadlines and prices of projects in complex defense systems. It is possible to not loose the investments made in legacy systems (e.g.: old aircraft models) written in FORTRAN language but that are validated already.

Based in our experience in projects at EMBRAER Empresa Brasileira de Aeronáutica S. A. and INPE National Institute for Space Research, we present a brief description of simulations environments used in development of spacecraft, aircraft (civil and military) and defense systems.

Spacecraft Simulation Environment

A general overview of a spacecraft simulation environment for research and development is shown in Figure 1.

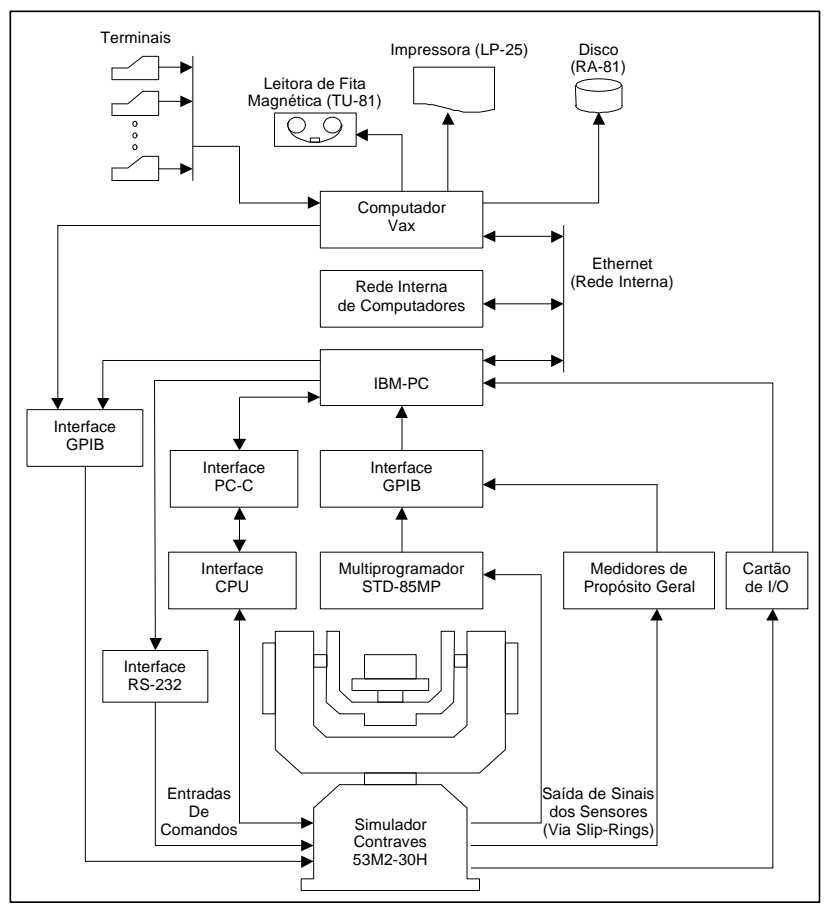

\section{Figure 1- General overview of a spacecraft simulation environment.}

Such environment may include various physical simula tion and tests facilities, using many dedicated softwares (built in Assembler, Basic, Fortran, C, etc.) to drive specific hardwares (Sun simulator, Earth simulator, 1 axis air table, 3 axis air table, controlled rotation table, Contraves.53MH2 dynamic simulator, etc.).

It may also include various computational simulation and tests facilities, using many general purpose modeling, identification and simulation environments (like Matlab/Simulink, MatrixX/AutoCode, STK Tool Kit, Constellation, etc.) used in research works (Scientific Initiation Reports, Master Dissertations, Doctoral Theses, etc.) and after, in project works with gains in productivity, correction, testability, etc. 


\section{Aircraft Simulation Environment}

A general overview of a (civil or military) aircraft simu lation environment for research and development is shown in Figure 2.

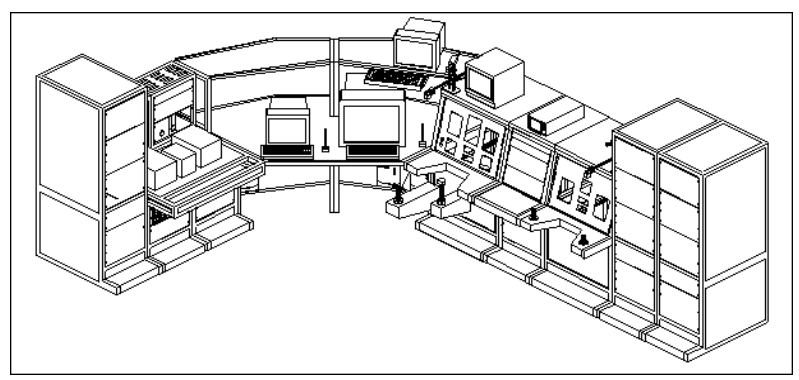

Figure 2 - General overview of an avionics integration station.

Such environment may include various physical simula tion and tests facilities, using many dedicated softwares (built in Assembler, Basic, Fortran, C, etc.) to drive specific hardwares (displays, cockpits, Rigs, IronBirds, etc.) in virtual or real time.

It may also include various computational simulation and tests facilities, using many general purpose modeling, identification and simulation environments (like Matlab/Simulink, MatrixX/AutoCode, VAPS, STAGE, Constellation, etc.) used in research and development works and after, in project works with gains in productivity, correction, testability, etc.

\section{Defense Systems Simulation Environment}

A general overview of a complex defense system simulation environment for research and development based in the HLA is shown in Figure 3:

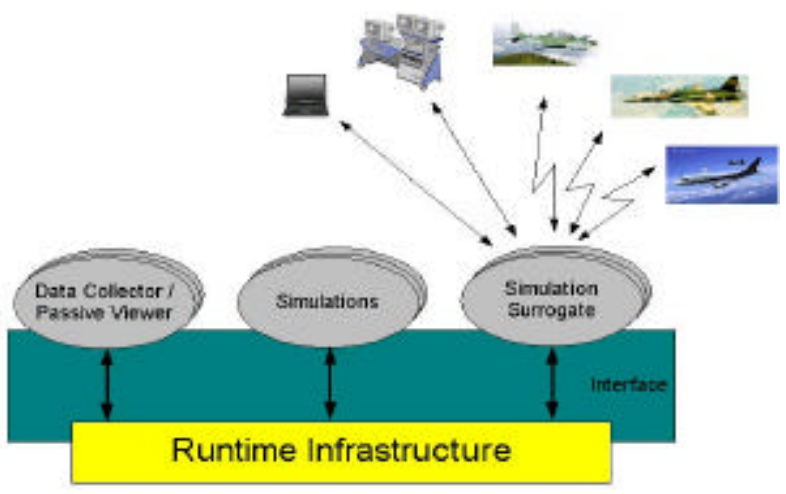

Figure 3 - Generic defense system based on HLA architecture.
Such environment includes and interconnects the elements of the three main categories possible in a simulation environment/situation: Data Collectors/ Passive Viewers (Information Monitors), Simulations; and Simulation Surrogates/Real Components. It allows the three main categories possible for simulations according to the DoD: constructive, virtual, or live.

It may include various physical simulation and tests facilities, using many dedicated softwares (built in Assembler, Basic, Fortran, C, etc.) to drive specific hardwares (displays, cockpits, RIGS, IronBirds, etc.).

It may also include various computational simulation and tests facilities, using many general purpose modeling, identification and simulation environments (like Matlab/Simulink, MatrixX/AutoCode,, VAPS, STAGE, Constellation, etc.) used in research and development works and after, in project works with gains in productivity, correction, testability, etc.

\section{REASONS FOR USING THE HLA}

Day by day the defense systems become more and more complex. As an example it may contain, at least, the following types of players: Airborne Early Warning and Control (AEW\&C) aircraft platform with highperformance multimode active radar mission system, including an onboard command \& control and advanced data-link suite; Remote Sensing and Surveillance Aircraft (RS/SA) providing real-time and near real-time imagery and electronic intelligence over ground objectives; Powerful Remote Sensing Maritime Patrol (RS/MP) up to complex and demanding antisurface/submarine capabilities; fighters with complex avionic and weapon system; cargo and refueling aircrafts; many types of ground support equipment including MPS, AACMI, DVDS, etc.; spacecraft for imaging and communications, datalink system connecting all players to each other and to with the ground infrastructure; everything supported by a very well coordinated and complex C4I 2 system.

The system is developed to work and interact with many others players in real world or in the synthetical environments. To fulfill its functionality all operators must be prepared to use it as much as possible. The simulation is not only the essential tool for development a system like this, but to prepares it for readiness and effectiveness. The reuse and interoperability is the key for timely and cost effective development. DoD has been improving their own year by year as the experiment presented in Torpey et alli ${ }^{6}$. 
The reasons for using the HLA become more evident the more complex and complete is the system to be simulated and the produtivity chain of the company that makes it. Despite less visible, it becomes even more important for the development of big projects distributed in diverse plants and even in different countries.. Its importance grows even more if we consider the possibility or the need of integration of the diverse projects under the sphere of a country or a community (ex. future air traffic control systems). To facilitate the comprehension, lets analyse the HLA advantages subdivided in the following aspects (even though a project could not include them all):

Standard Architecture and the De Facto Standards:

With the essential preoccupation of reducing costs, the DOD supported for a decade the research and development of a modeling and simulation architecture: the HLA. This architecture became the IEEE 1516/00 in 2000, to be adopted in USA and in Europe by the producers/buyers of defense systems that use models and simulations.

Besides and before HLA, a series of official or de facto standards have been collaborating in the reduction of costs, time and risks in similar projects, like: OpenGL (Human-Machine Interfaces-HMI), Open Flight (Flight), DTED (Terrain), etc.

\section{Technical Advantages}

As a consequence of the time and process used in its development, the HLA has the following main characte ristics/advantages:

- Interoperability;

- Reuse;

- Connectivity;

- Open standard architecture;

- Infrastructure that allows information monitoring, connections among simulations, and connections to real participants;

- Maturity - it was based in the experience of the American defense community, with the participation and discussion of the diverse groups involved in such subjects;

- Adherence to legacy projects and to object oriented projects (distributed or not);

\section{Reuse of Environments}

The adoption of a unique architecture allows the reuse of integration and tests environments in different programs of a company. Consequently, we obtain a reduction of time and costs and an improvement of the processes of the company. Beyond the processes directly tied to tests of complex systems, processes in other areas are improved, and even new business opportunities are created due to the changes mentioned ahead.

Redistribution of Time among the Design Phases Today, it is notorious the increase in volume and complexity of embedded flight softwares or engineering systems support softwares, particularly in the aerospace engineering and in defense systems. The use of advanced technologies has provoked a consequent displacement of the hours in each phase of a program. The time spent with ground or air tests has increased in two aspects: i) in the absolute value, due to the increase of complexity; and ii) in the relative value, due to the reduction in time of the other phases. For example, in the case of the Joint Simulation Force-JSF, the time for design and codification of the Human-Machine Interface-HMI was practically reduced to the managerial safety margin, with the use of display specifications done in VAPS.

\section{Complexity of Tests}

The complexity of tests has combinatorial growth (much larger than linear growth), with/due to the growth of the number and complexity of the functionalities. Consequently, the development time for test environments may become the product noncompetitive if such environments or their components are not reused. But for their reuse, it is necessary the adoption of a common architecture.

\section{Tests of Defense Systems}

The current defense systems became very complex with the integration of data links and with the incorporation of terrestrial networks with different types of ground stations for a vast range of applications. As the last instance of sophistication, we have the complex Command, Control, Computer, Communication, Intelligence and Information-C4I2 environments. Given the high degree of complexity, risk and cost, great part of the functionalities can only be tested or demonstrated on ground or in Synthetic Environments-SE.

\section{Costs of the Tests}

Beyond the situations where flight tests are impracticable, the impact of the number of flights necessary for tests is large in complex defense systems. The number of tests for one or even more flights becomes a fundamental control item in the costs of a 
program. A greater number of tests with more complexity increase the probability of repeating a flight test or part of it. Immediately, this makes it necessary a more elaborate test plan and, consequently, a better preparation of the flight tests in ground.

\section{Preparation for Tests}

The preparation in ground, for flight tests of complex defense systems demand simulation environments including the aircraft and its environment. The flight test engineers shall use these environments to reproduce and analyze the most important phases, and to brief and debrief the test team, including the test pilots. The "a priori" simulation of the tests to be done is an essential tool for training the team and pilots and consequent improvement in the uses of the flight tests. The aspects of test safety and reliability must also be considered.

\section{Test Environments}

The test environments become not useful or with prohibitive costs without the reuse of components and a common architecture with other devices of a defense system: diverse ground stations, simulators, trainers, C4I2 environments, etc.

Defense systems have diverse partners and suppliers even from different countries. The best form if not the only form to use and reuse a common architecture and/or common components is the adoption of a standard architecture, and even standards for subcomponents or sub-systems.

\section{$\underline{\text { Demonstrations }}$}

Integrated simulation environments can be used to demonstrate the technological capacity of a company or the meeting of requirements. Due to the high cost of flight tests, there are requirements whose verification can be substituted, in part or in the whole, by demonstrations and tests in synthetic environments. Their percentage becomes large with the certification of the models through processes of Verification, Validation and Accreditation-VVA.

\section{Certification}

Environments of such nature are indispensable tools in the processes of certification of aircrafts. The use of a common architecture reduces their time and costs.

\section{Marketing}

The marketing of a complex defense system demands the use of complex tools. How we convince a potential customer of the advanced technological content of a product and its efficacy, without using marketing tools void of technology? What would be the cost of developing such marketing tools without a good basis? How can we demonstrate the quality of a complex defense system without simulations?

\section{Pre-designs}

The area of pre-designs is fundamental for any company in the aerospace and defense markets. At EMBRAER this was emphasized with the creation of a specific area with people of great experience. Which tools are available today? Which ones will be available in the future? What would be the impact of simulation environments including systems, displays, aircrafts and their environments, terrains, etc. if they become available?

\section{Training}

The quality of civil training improves with products of greater quality in the area of simulators and with products of lower cost for simpler devices. These simpler devices then become largely used even by small aircrafts. With the sophistication of the military aircrafts and respective defense systems, these training devices become fundamental. The question of cost of training a pilot in flight or in simulators adds up to the question of efficacy of such trainings, especially when treating of aircrafts with complex HMI and integrated with large defense systems. In the currently available defense systems, the new capacities born from the technological advances can only be trained with efficacy in integrated simulated environments: the Synthetic Environments-SE. For training in C4I2 environments this necessity becomes even stronger.

\section{Air Traffic Control and Management System (ATCMS)}

Today, a new ATCMS is under strong development. It will allow a large increase in the air traffic capacity, allowing the pilot to change its own route. This will cause a fantastic expansion in the market of air traffic control, very important for the aerospace companies since it involves interconnections of satellites, aircrafts, airports, etc. How can we research and develop products and services in this area without synthetic simulated environments? The flight control towers will soon be substituted by equivalent synthetic environments integrated to data received in real time from aircrafts and ground vehicles. Is it possible to develop an industry in this direction without standards? A proposal for such a research and development environment based in the HLA is presented by Roza and $\mathrm{Gool}^{5}$. 


\section{CONCLUSIONS}

The simulation environments already are constant tools in the design of satellites, civil and military aircrafts, defense systems and some air traffic control systems. In all companies of the aerospace area there is a set of tools, models and simulations supporting the systems developed and under development. Occasional changes will always be associated to risks and costs, mainly current costs (related to actually making it work). However, the new projects are more and more complex and demand more and more sophisticated environments for analysis, design, development, integration, tests, certification, training, marketing, support and maintenance. Simulations are a more and more essential and integrating element in all these environments during a project, and even more, among the diverse projects of a company or group of companies. The "adoption of a standard for simulation environments" appears as a necessity stronger than ever. Considering the analysis of costs, this is a decision typical of the whole company, but not of a specific project, since its benefits are global. In taking the opposite decision, i.e., of the "no adoption of a standard for simulation environments ", it would be fundamental to estimate the costs of it, as prescribed by the soundest economic theories, despite these are not familiar to technical communities. So, considering the diverse reasons explained in this work, the standard for simulations suggested is the HLA.

\section{REFERENCES}

[1] Institute for Electrical and Electronic Engineers (IEEE). "IEEE Standard for Modeling and Simulation (M\&S) HIGH Level Architecture (HLA) - Framework and Rules“. IEEE Press, New York, NY, USA, 2000, 21 p.

[2] Institute for Electrical and Electronic Engineers (IEEE). "IEEE Standard for Modeling and Simulation (M\&S) High Level Architecture (HLA) - Federation Interface Specification". IEEE Press, New York, NY, USA, 2000, 129 p.

[3] Institute for Electrical and Electronic Engineers (IEEE). "IEEE Standard for Modeling and Simulation (M\&S) HIGH Level Architecture (HLA) - Object Model Template (OMT) Specification". IEEE Press, New York, NY, USA, 2000, 466 p.

[4] Kuhl, F. K.; Weatherly, R. and Dahmann, J. "Creating Computer Simulation Systems". Prentice Hall, Englewood Cliffs, USA, 2000. 212 p.

[5] Roza, M. and Gool, P.V., 2000, "Simulating Free Flight in HLA Federations", Proceedings of AIAA 2000 GNC, AFM, and MST Conferences, Denver, $\mathrm{CO}, \mathrm{USA}$.

[6] Torpey, M., Helfinstine, B. and Evans, J, 2002, "Reflections on Building the Joint Experimental Federation", Proceedings of IITSEC 2002 Conference, Orlando, FL, USA. 